STRUCTURAL BIOLOGY

ISSN 2059-7983

\title{
Direct-methods structure determination of a trypanosome RNA-editing substrate fragment with translational pseudosymmetry
}

\author{
Blaine H. M. Mooers*
}

Department of Biochemistry and Molecular Biology, and Stephenson Cancer Center, University of Oklahoma Health Sciences Center, 975 NE 10th Street, BRC 466, Oklahoma City, OK 73104, USA. *Correspondence e-mail: blaine-mooers@ouhsc.edu

Received 3 October 2015

Accepted 19 January 2016

Edited by Z. Dauter, Argonne National Laboratory, USA

Keywords: RNA structure determination; Patterson analysis; helical symmetry; ab initio phasing; noncrystallographic symmetry.

PDB references: RNA (32-mer), 5da6; RNA hairpin, $5 \mathrm{~d} 99$

Supporting information: this article has supporting information at journals.iucr.org/d
Using direct methods starting from random phases, the crystal structure of a 32base-pair RNA (675 non-H RNA atoms in the asymmetric unit) was determined using only the native diffraction data (resolution limit $1.05 \AA$ ) and the computer program SIR2014. The almost three helical turns of the RNA in the asymmetric unit introduced partial or imperfect translational pseudosymmetry (TPS) that modulated the intensities when averaged by the $l$ Miller indices but still escaped automated detection. Almost six times as many random phase sets had to be tested on average to reach a correct structure compared with a similar-sized RNA hairpin (27 nucleotides, 580 non-H RNA atoms) without TPS. More sensitive methods are needed for the automated detection of partial TPS.

\section{Introduction}

Automated structure determinations by direct methods of equal-atom proteins (i.e. atoms lighter than calcium) with 1000 non-H protein atoms have been achieved when starting from random phase angles (i.e. ab initio direct methods), when using dual-space methods and when the diffraction data extend to atomic resolution (Sheldrick, 1990; Morris \& Bricogne, 2003; Langs \& Hauptman, 2011; Giacovazzo, 1998, 2014). These constraints are relaxed when calcium or heavier atoms are present, when Patterson superposition methods are used or when Patterson methods and heavy atoms are used together (Burla et al., 2006; Caliandro et al., 2007, 2008; Mooers \& Matthews, 2004, 2006). We found only one report of $a b$ initio direct methods being successfully applied to an unknown RNA molecule (Safaee et al., 2013). Success in direct-methods structure determination could be expected to be easier with nucleic acids than with proteins because the $\mathrm{P}$ atoms in the backbone of RNA are electron-dense, even though they are sometimes in two alternate conformations (Luo et al., 2014), and because the $\mathrm{P}$ atoms occur at a higher frequency $(\sim 1$ in 20$)$ in nucleic acids than $S$ atoms occur in proteins (1 in 100-300; Ramagopal et al., 2003). On the other hand, translational pseudosymmetry (TPS) caused by helices longer than one turn may inhibit structure determination by direct methods because the internal symmetry violates the assumption that the atoms in the asymmetric unit are randomly distributed. This idea is supported by many reports of TPS hindering the direct-methods structure determination of small-molecule crystal structures and the molecularreplacement structure determination of proteins (Dauter et al., 
Table 1

Data collection and processing for dsRNA (PDB entry 5da6).

Values in parentheses are for the outer shell.

\begin{tabular}{ll}
\hline Diffraction source & Beamline 7-1, SSRL \\
Wavelength (̊) & 0.97946 \\
Temperature (K) & 100 \\
Detector & ADSC Quantum 315r \\
Space group & $R 32: H$ \\
$a, b, c(\AA)$ & $42.89,42.89,266.94$ \\
Mosaicity $\left({ }^{\circ}\right)$ & 0.22 \\
Resolution range $(\AA)$ & $36.79-1.05(1.10-1.05)$ \\
Total No. of reflections & $355361(41841)$ \\
No. of unique reflections & $41841(6468)$ \\
Completeness $(\%)$ & $99.9(99.9)$ \\
Multiplicity & $7.9(6.8)$ \\
$\langle I / \sigma(I)\rangle$ & $20.1(2.27)$ \\
$R_{\text {r.i.m. }}$ & $0.057(0.440)$ \\
Overall $B$ factor from Wilson plot $\left(\AA^{2}\right)$ & 11.3 \\
\hline
\end{tabular}

2005). The role of TPS in phasing has been explored many times in chemical crystallography and is a current interest in biological crystallography (Hauptman \& Karle, 1959; Böhme, 1982; Gramlich, 1984; Cascarano et al., 1985, 1987; Fan, Qian et al., 1988; Fan, Yao et al., 1988; Zwart et al., 2008; Read et al., 2013). The rational dependence of the atoms related by TPS leads to sets of strong reflections and weak reflections. Most of the phase relationships depend on strong reflections if the presence of TPS is ignored. The weak reflections can be used to form separate phase relationships (Cascarano et al., 1988a,b; Fan, Qian et al., 1988; Fan, Yao et al., 1988). Rotational pseudosymmetry in crystal structures of short dsRNAs has been reported (Kondo et al., 2008) and the prospects for direct methods with oligonucleotides shorter than one helical turn have been explored (Hubbard et al., 1994), but we know of no published applications of direct methods to nucleic acids with TPS present. The most common TPS in protein crystals involves two molecules in the asymmetric unit. Sometimes TPS is found within a single protein; three of 1007 protein superfamilies have internal TPS (Myers-Turnbull et al., 2014). In contrast, RNA double helices longer than one helical turn could have imperfect TPS caused by the helical repeats. This TPS could restrict success in direct methods to RNAs of one helical turn in length or shorter. Previous nucleic acid structures determined $a b$ initio by direct methods have been one helical turn long or shorter (Egli et al., 1998; Han, 2001; Safaee et al., 2013; Luo et al., 2014).

We tested the idea that SIR2014 could still determine the structure of a dsRNA with imperfect TPS by ab initio direct methods without detecting the TPS. We compared the directmethods structure determination of a double-stranded RNA (dsRNA; 32 base pairs, one strand in the asymmetric unit, 675 non-H atoms, two levels of imperfect TPS) with that of a single-stranded RNA (ssRNA) hairpin (27 nucleotides, one strand in the asymmetric unit, 580 non-H RNA atoms, no TPS). The dsRNA is a pathological case for ab initio structure determination in the presence of TPS and the hairpin is a case for $a b$ initio structure determination by direct methods in the absence of TPS. The $a b$ initio structure-determination experiments were performed with the direct-methods program SIR2014, which uses dual-space methods to attempt structure determination from random phases. Owing to the stochastic nature of the phasing process (i.e. starting from different sets of random phases in each trial), the number of failed trials before success in one phasing experiment says little about the next phasing experiment that tests a different series of random phase sets. Therefore, a large number of phasing experiments were conducted to obtain the empirical probability mass function (pmf) of success with each data set. The pmf for the dsRNA was broader than that for the hairpin and the mean number of trials was almost six times larger. To investigate this difference, we compared the intensity distributions, Patterson maps, the translation vectors used to shift misplaced trial structures and the effect of removing the strongest reflections on success in structure determination. The presence of TPS enhanced the strong intensities and made the loss of the strongest intensities a larger problem. Our results should appeal to workers interested in phasing methods, RNA crystallography or both.

\section{Materials and methods}

\subsection{Construct design, crystallization and data collection}

The design, crystallization, $\mathrm{X}$-ray diffraction data collection, structure determination and structure description of the hairpin RNA (PDB entry 3dw4) have previously been published (Olieric et al., 2009). The related structure factors were retrieved from the Protein Data Bank. This hairpin is from the sarcin/ricin domain of the Escherichia coli 23S RNA (Olieric et al., 2009). The same experimental aspects of the dsRNA will be described in detail elsewhere, so they are only summarized here. Two 16-base pair U-helix domains from a RNA-editing substrate in trypanosomes were fused head-tohead to promote duplex formation by the $3^{\prime}$ tail of 16 Us that would otherwise form a random coil with unstacked bases in solution at room temperature (Mooers \& Singh, 2011). The fusion RNA was made by phosphoramidite chemistry and was gel-purified to single-nucleotide resolution (Dharmacon, GE Healthcare). Crystals were grown at room temperature from $50 \mathrm{~m} M$ sodium cacodylate $\mathrm{pH} 6.5,20-50 \mathrm{~m} M \mathrm{MgCl}_{2}, 1-2 M$ lithium sulfate. The crystals were cryoprotected by passage through 1.9, 2.4 and $2.9 \mathrm{M}$ sodium malonate $\mathrm{pH}$ 6.0. (There was no evidence of arsenic in the X-ray fluorescence scans of similarly treated crystals because the sodium malonate had displaced the cacodylate molecules in the crystal.) X-ray diffraction data were collected on beamline 7-1 at SSRL with $0.979 \AA$ wavelength radiation and an ADSC Quantum 315r detector. The diffraction data were collected at four distances between the detector and the crystal to properly measure the very strong reflections at medium resolution associated with the base stacking in the RNA. The long $c$ edge of the unit cell was manually aligned within $40^{\circ}$ of the rotation axis of the crystal to avoid spot overlap at high resolution. About 40 crystals with a longest dimension of $0.2-0.4 \mathrm{~mm}$ were screened for diffraction quality. Most crystals diffracted X-rays to between 1.4 and $1.2 \AA$ resolution, but one crystal diffracted $\mathrm{X}$-rays to $1.05 \AA$ resolution and was selected for data 
collection. The diffraction data were processed with iMOSFLM (Battye et al., 2011) and SCALA (Evans, 2006). Data-collection statistics are reported in Table 1.

\subsection{Direct-methods structure-determination experiments}

The merged native data for the dsRNA were used with the computer program SIR2014 (Burla et al., 2015) running on individual central processing units (CPUs) on a Xeon64 octa-core Linux cluster in the Oklahoma Center for Supercomputing Education and Research (OSCER) at the University of Oklahoma. Each CPU executed an independent experiment that tested up to 600 different sets of random phases. Each structure-determination trial started with a different set of phases. The phases were pseudorandom numbers that could be recreated by specifying the index of the phase set. The SIR2014 code was not parallelized for the execution of one phasing trial on multiple CPUs or on graphical processing units. The modern direct-methods (MDM) phasing protocol in SIR2014 was used with its default parameters. The $R E L A X$ procedure was available to all phasing trials; this protocol shifted to the correct origin promising phase sets that were developing near the wrong origin (Burla et al., 2000; Caliandro et al., 2007). To find the shift vector, the diffraction data were expanded to $P 1$. After the shift vector was located in $P 1$, the program returned to the original space group. The same structure-determination procedure was used with the hairpin RNA.

\subsection{Automated model building and refinement}

The first correct $a b$ initio phases for the dsRNA and the hairpin RNA were used in automated model building with Nautilus (Cowtan, 2014). The models from Nautilus were corrected manually using Coot (Emsley et al., 2010). The RCrane plugin for Coot was used with the hairpin RNA, which required extensive correction owing to the presence of several non-Watson-Crick base pairs (Keating \& Pyle, 2012). The refinement of each model was started at the resolution limit using stereochemistry restraints derived from atomic resolution crystal structures of nucleotides, PHENIX and all of the diffraction data (Parkinson et al., 1996; Adams et al., 2010). The REEL program within PHENIX was used to generate stereochemical restraints for the $\mathrm{O} 2^{\prime}$-methyluridine found at

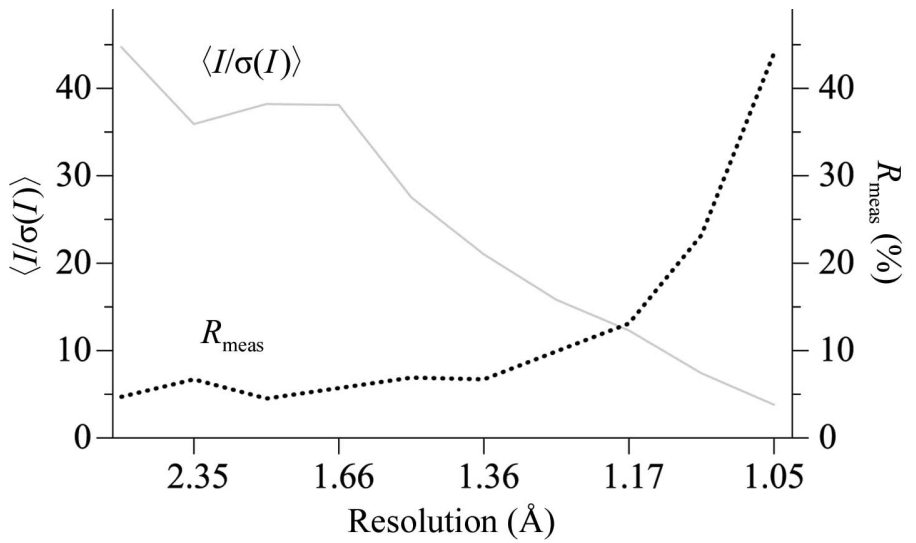

$(a)$

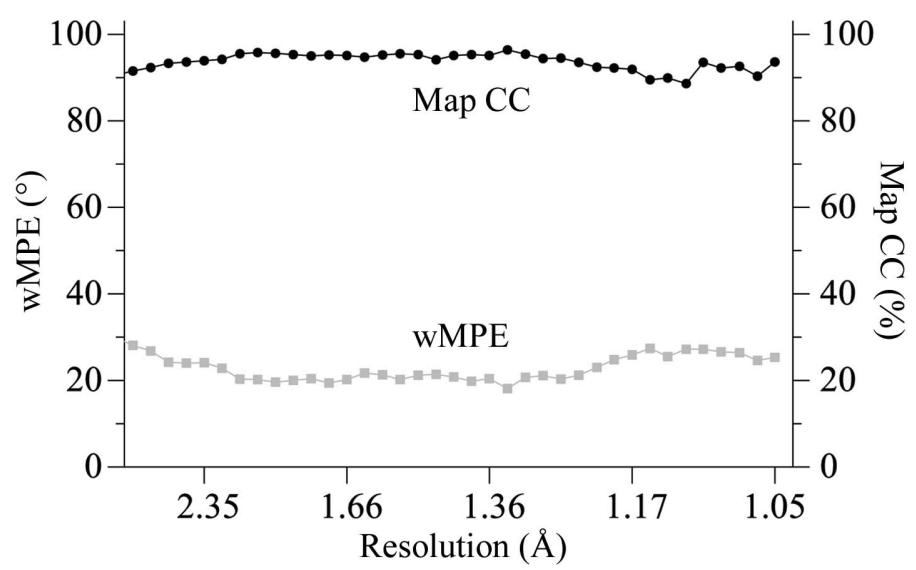

(c)

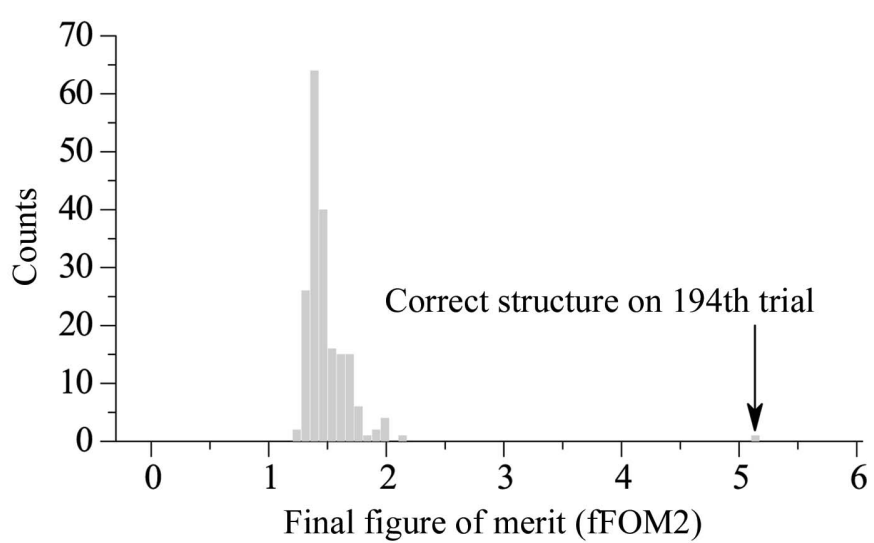

(b)
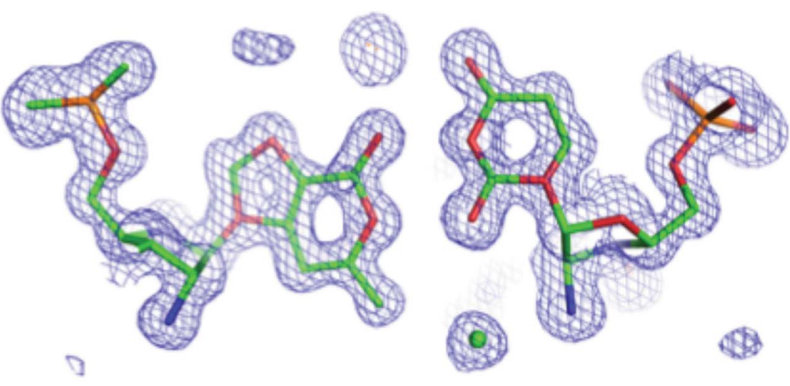

(d)

Figure 1

Initial $a b$ initio structure determination of the 32-nucleotide dsRNA by direct methods using SIR2014 (PDB entry 5da6). (a) Data quality as indicated by $R_{\text {meas }}$ and the $\langle I / \sigma(I)\rangle$ signal-to-noise ratio. (b) The distribution of the final figure of merit (fFOM2) for the 194 phasing trials in the first experiment. (c) The weighted mean phase error (wMPE) versus resolution and the map correlation coefficient (mapCC) versus resolution for the winning phase set in (b) (Lunin \& Woolfson, 1993). The final refined model served as the source of the 'true' phases. (d) $F_{\mathrm{o}} \exp (S I R 2014$ phases) electron-density map for dsRNA with the model from automated peak picking without knowledge of the RNA stereochemistry. The atom types are colored as follows: carbon, green; nitrogen, blue; oxygen, red; phosphorus, orange. The map was rendered with PyMOL. 
Table 2

Structure refinement for structures determined ab initio with SIR2014.

Values in parentheses are for the outer shell.

\begin{tabular}{|c|c|c|}
\hline & $\begin{array}{l}\text { U-helix dsRNA } \\
\text { (PDB entry 5da6) }\end{array}$ & $\begin{array}{l}\text { Hairpin RNA } \\
\text { (PDB entry 5d99) }\end{array}$ \\
\hline Resolution range $(\AA)$ & $36.8-1.05(1.10-1.05)$ & $21.0-0.97(0.98-0.97)$ \\
\hline Completeness (\%) & $99.6(99.1)$ & $99.9(98.6)$ \\
\hline$\sigma$ Cutoff & 2.0 & 2.0 \\
\hline No. of reflections, working set & 42604 & 38814 \\
\hline No. of reflections, test set & 2337 & 2029 \\
\hline Final $R_{\text {cryst }}$ & 0.1301 & 0.105 \\
\hline Final $R_{\text {free }}$ & 0.1568 & 0.120 \\
\hline Cruickshank DPI $\dagger$ & 0.025 & 0.018 \\
\hline \multicolumn{3}{|l|}{ No. of non-H atoms } \\
\hline RNA & 675 & 580 \\
\hline Ligand & 1 & 14 \\
\hline Water & 236 & 183 \\
\hline \multicolumn{3}{|l|}{ R.m.s. deviations } \\
\hline Bonds $(\AA)$ & 0.005 & 0.006 \\
\hline Angles $\left(^{\circ}\right)$ & 1.0 & 1.2 \\
\hline Average $B$ factor for RNA $\left(\AA^{2}\right)$ & 13.98 & 10.78 \\
\hline MolProbity clash score & 0.0 & 0.0 \\
\hline
\end{tabular}

$\dagger$ Computed using Online_DPI (Kumar et al., 2015).

position 2650 in the hairpin RNA (Moriarty et al., 2009). The refinements were initiated with isotropic atomic displacement parameters (ADPs) and no $\mathrm{H}$ atoms. Large drops in $R_{\text {free }}$ on the change to anisotropic ADPs justified replacing the isotropic ADPs with anisotropic ADPs. Likewise, smaller but still significant drops in $R_{\text {free }}$ warranted the addition of $\mathrm{H}$ atoms. The final refinement statistics are reported in Table 2. The final structures (dsRNA, PDB entry 5da6; redetermined hairpin RNA, PDB entry 5d99) have been deposited in the Protein Data Bank and the Nucleic Acid Database (Berman et al., 2000).

\section{Results}

We compared the structure determinations of dsRNA with three helical turns and of a hairpin with one helical turn and thus no TPS. The diffraction data for the dsRNA were $99 \%$ complete (Table 1) and had a resolution limit of $1.05 \AA$ (Fig. 1a). The native Patterson map showed evidence of TPS (Fig. 2). The hairpin RNA was the closest in size to the $32 \mathrm{nt}$ RNA of the available RNA structures with diffraction data at similar resolution. Its diffraction data were nearly complete, and the structure lacked calcium or heavier atoms. Next, we describe the initial structure determination of the dsRNA. The same structure-determination procedure was used with the data from the $27 \mathrm{nt}$ hairpin RNA. We compared the distribution of the number of failed trials before a correct structure for the dsRNA and the hairpin, and found a large difference. We also found differences in the distributions of the intensities and of the vectors used to shift misplaced trial structures. In addition, we found a difference in the sensitivity to the removal of the strongest reflections. The details of the structure of the dsRNA are irrelevant to the central question of this paper and will be described elsewhere. Because each case has a sample size of one, the results reported below cannot be used

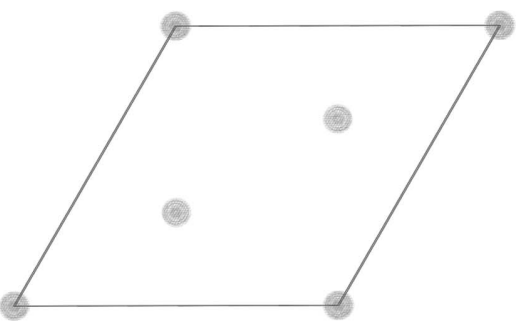

(a)

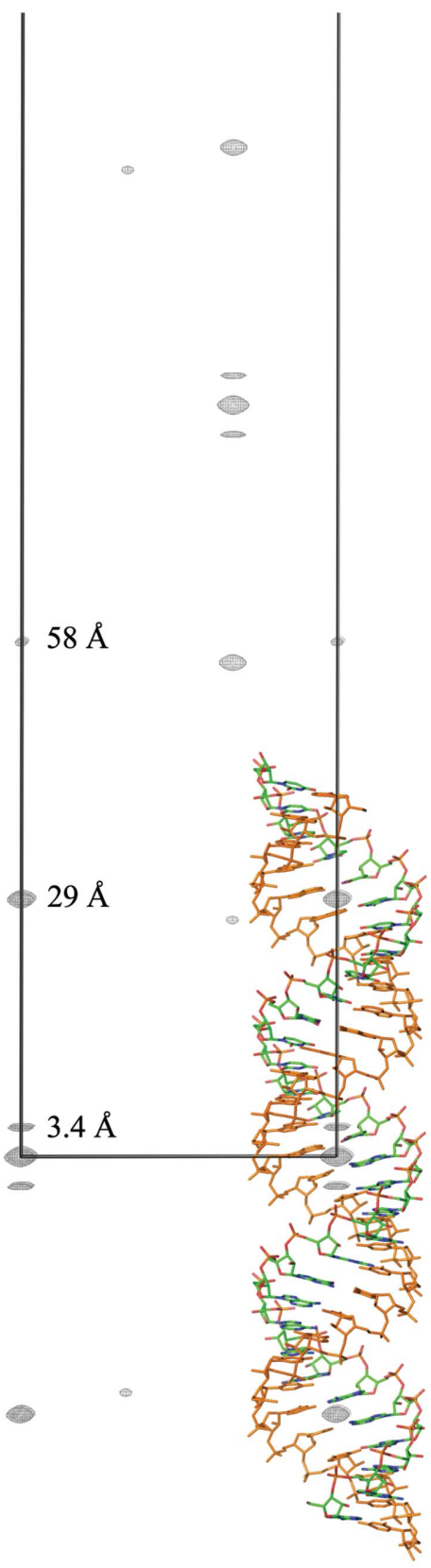

(b)

Figure 2

Native Patterson map of the dsRNA obtained with $1.05 \AA$ resolution diffraction data (PDB entry 5da6). (a) Map at the $w=0$ level contoured at the $12 \sigma$ level. (b) Map at the $u=0$ level contoured at the $12 \sigma$ level. A stick model of the biological unit without the solvent is overlaid on the unit-cell origin. The single-colored strand was generated by crystallographic symmetry. The off-origin peak at $29 \AA$ corresponds to the length of one helical turn and the peak at $58 \AA$ corresponds to the length of two helical turns. 
to make inferences about the ease of structure determination by direct methods with diffraction data from other RNAs.

\subsection{TPS in the asymmetric unit}

The $32 \mathrm{nt}$ dsRNA was a head-to-head fusion of two Uhelices from a RNA-editing substrate from trypanosomes (Mooers \& Singh, 2011). The $3^{\prime}$ half of the fusion RNA consisted of 16 consecutive Us that represented the U-tail of the guide RNA. This tail formed ten A-U Watson-Crick base pairs and six G-U wobble base pairs with the $5^{\prime}$ half of the fusion RNA. The $5^{\prime}$ half represented the purine-rich preedited mRNA. The RNA was one base pair short of the 33 base pairs required for three helical turns in A-form RNA (11 base pairs per turn). Three double helices stacked end-on-end along the $c$ edge of the $R 32: H$ unit cell. One strand was in the asymmetric unit (colored by atom type in Fig. 2b). The base pairs were inclined by about $16^{\circ}$ with respect to the $c$ edge of the unit cell. Strong peaks appeared $3.4 \AA$ from the origin in the native Patterson map; these peaks corresponded to parallel, interatomic vectors between adjacent base pairs (Fig. 2). The interatomic vectors between a base pair and its next-nearest neighbor were much weaker. A peak with a height $57.2 \%$ of that of the origin peak was located at a distance of $29 \AA$ from the origin along the $w$ edge of the Patterson map. This distance corresponded to the length of one helical turn (Fig. 2b). Translation vectors between atoms in turns 1 and 2 (r.m.s.d. of $1.3 \AA$ for backbone atoms only) and between atoms in turns 2 and 3 (r.m.s.d. of $1.3 \AA$ for backbone atoms) (Fig. 3a) gave this peak a double weight. A smaller peak along $w$ at $58 \AA$ (Fig. $2 b$ ) from the origin was caused by the vectors between turns 1 and 3 (r.m.s.d. of $1.7 \AA$ for backbone atoms). These longer vectors were half of the number of the vectors that made the higher peak and lead to a second off-origin peak less than half the height of the first peak. The dsRNA crystal structure with the second turn deleted gave a calculated Patterson map that lacked the first peak. Likewise, the crystal structure with the third turn deleted gave a calculated Patterson map that lacked the second peak. These calculated maps validate our interpretation of the native Patterson map. The TPS was too imperfect to be detected automatically by the algorithm used by SIR2014.

The hairpin had 11 base pairs in the stem, two unpaired bases in the hairpin loop and two unpaired bases at the termini (Fig. 3b). The stem was not longer than one helical turn, so it lacked TPS caused by helical turns. The stem of the hairpin was aligned parallel to the diagonal of the $\mathbf{a} \times \mathbf{b}$ face of the unit cell (Fig. 4a), so the normal vectors of many of the base-pair planes were parallel to the diagonals on the $\mathbf{a} \times \mathbf{b}$ face of the tetragonal unit cell. These interatomic vector lead to accumulation of Patterson density along these diagonals (Fig. 4). No peaks of $>5 \sigma$ were found beyond the peaks owing to the adjacent base pairs, so the Patterson of the hairpin gave no evidence of TPS from helical repeats.

\subsection{Initial $a b$ initio structure determination of the dsRNA}

To determine the structure of the dsRNA, we used an almost complete diffraction data set. This data set had a resolution limit of $1.05 \AA$ and a Wilson $B$ factor of $10.6 \AA^{2}$ (Table 1; Fig. 1a). We used SIR2014 v.7 for structure determination. We used the modern direct-methods (MDM) protocol in SIR2014, which starts with the atomic composition of the RNA and a set of random phases. SIR 2014 converted the observed structure factors $(F \mathrm{~s})$ into normalized structure factors $(E \mathrm{~s})$. The 3188 largest $E \mathrm{~s}\left(\left|E_{\min }\right|=1.716\right)$ were used to develop the phase relationships. SIR2014 used 300000 structure invariants with an extended tangent formula to refine the phases of the strongest Es (Burla et al., 2013). The phases were then extended and refined in real space by density modification. SIR2014 used the default parameter values in the directspace refinement (DSR) module.

SIR2014 used the global phasing statistic called the 'final figure of merit 2' (fFOM2) to assess the promise of a phase set:

$$
\mathrm{fFOM} 2=\mathrm{CC}(\text { all })_{\text {current }} \times \frac{\mathrm{RAT}_{\text {current }} \mathrm{CC}(\text { all })_{\text {current }} \mathrm{CC}(\text { large })_{\text {current }}}{\mathrm{RAT}_{\text {initial }} \mathrm{CC}(\text { all })_{\text {initial }} \mathrm{CC}(\text { large })_{\text {initial }}},
$$

$$
\mathrm{RAT}=\frac{\mathrm{CC}_{w, E}}{\left\langle E_{\text {calc }}^{2}\right\rangle_{\text {weak }}} .
$$

$\mathrm{CC}$ is the correlation coefficient between the observed and calculated normalized structure factors. The word 'all' means all of the normalized structure factors $E$; 'large' means the subset of reflections with the $70 \%$ largest $E$ s and 'weak' means

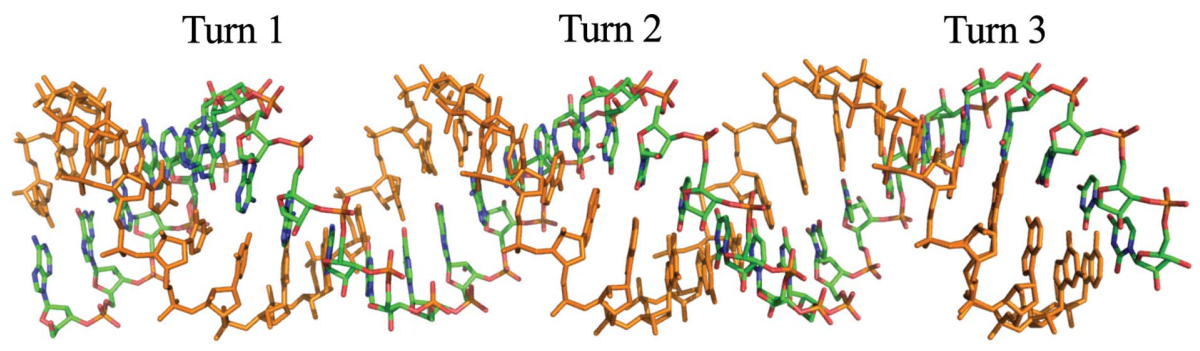

(a)

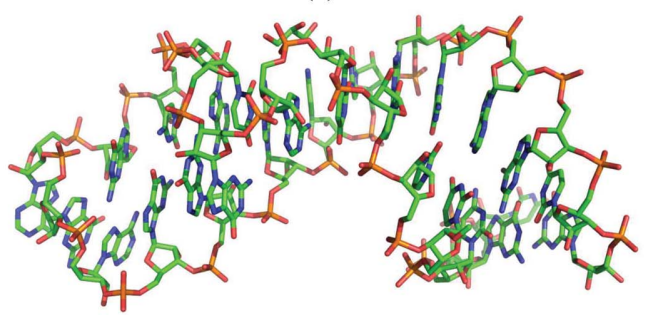

(b)

Figure 3

Comparison of the final structures of ( $a$ ) the dsRNA (PDB entry 5da6) and (b) the hairpin RNA (PDB entry $3 \mathrm{wd} 4)$. The single-colored strand in $(a)$ was generated by crystallographic symmetry. 
the subset of reflections with the $30 \%$ smallest $E s . C_{w, E}$ is the correlation coefficient between the largest $70 \%$ of observed $E$ s and the corresponding statistical weights. The $E_{\text {calc }}$ values are from the inverse Fourier transform of the current electrondensity map. RAT (2) is a global figure of merit used in past versions of SIR (Burla et al., 2002). $\left\langle E_{\text {calc }}^{2}\right\rangle_{\text {weak }}$ is the mean of the $30 \%$ of the Es with the weakest amplitudes.

When the fFOM2 for a trial was greater than 3.0, the trial was likely to be a success. SIR2014 wrote the phases and coordinates to files and then stopped. SIR2014 never used the remaining sets of random phases from the initial collection of 600. If the fFOM 2 remained below 3.0, SIR2014 abandoned the phase set. SIR2014 selected the next phase and repeated the structure-determination protocol. The default limit of 600 phase sets was used in each phasing experiment. If all 600 phase sets failed, SIR2014 stopped and we tallied the phasing experiment as a failure. The correct phase set or its opposite

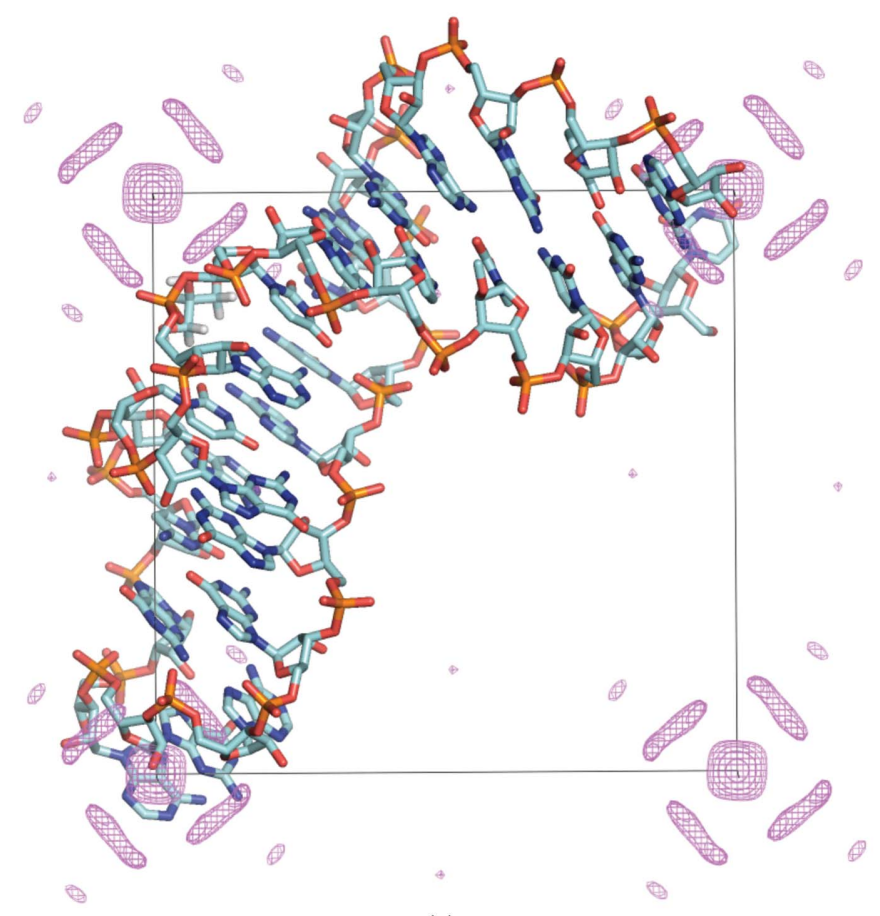

(a)

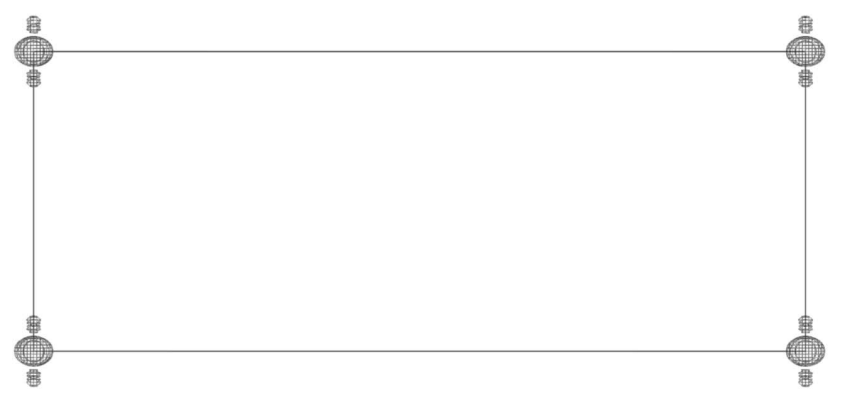

(b)

Figure 4

Native Patterson map generated from the $0.97 \AA$ resolution diffraction data of the hairpin RNA (PDB entry $3 w d 4)$. (a) Map generated with all of the data contoured at the $w=0$ level and contoured at the $3 \sigma$ level. $(b)$ The same map contoured at the $12 \sigma$ level. A stick model of the RNA (solvent is hidden) is shown at its position in the unit cell. hand was not distinguished by the fFOM2. Both hands were counted as successes because changing the hand of the phases is trivial.

The 194th random phase set for the dsRNA (Fig. 1b) gave the first value (5.115) for the fFOM2 that was greater than 3.0 (Fig. 2b). SIR2014 stopped after writing out the final coordinates and phases and left the remaining 406 phase sets untested. This 194th phase set had a low weighted mean phase error $\left(\mathrm{wMPE}=23.3^{\circ}\right.$; Fig. $\left.1 c\right)$ when compared with the final refined structure. The final trial was reached after 9 h 56 min on a single CPU at OSCER. One processor on a late-2013 MacBook Pro laptop computer with 16 GB of RAM took a similar amount of time to reach a correct structure.

The complete RNA strand and many solvent molecules appeared in the figure of merit (FOM)-weighted $F_{\mathrm{o}}$ map that was obtained with the ab initio phases from SIR2014 (Fig. 1d). SIR2014 placed atoms by peak picking and assigned atom types by peak height. The $31 \mathrm{P}$ atoms of the one strand in the asymmetric unit were assigned correctly, but the assignment of the light $\mathrm{C}, \mathrm{N}$ and $\mathrm{O}$ atoms had errors. Variation in the ADPs of $\mathrm{C}, \mathrm{N}$ and $\mathrm{O}$ atoms caused overlap in the peak heights for these atoms. For example, errors are obvious in the model of a G-U wobble base pair (Fig. 1d). Manual correction of this model was error-prone, so we replaced the SIR2014 model with a model built by Nautilus. We manually corrected the

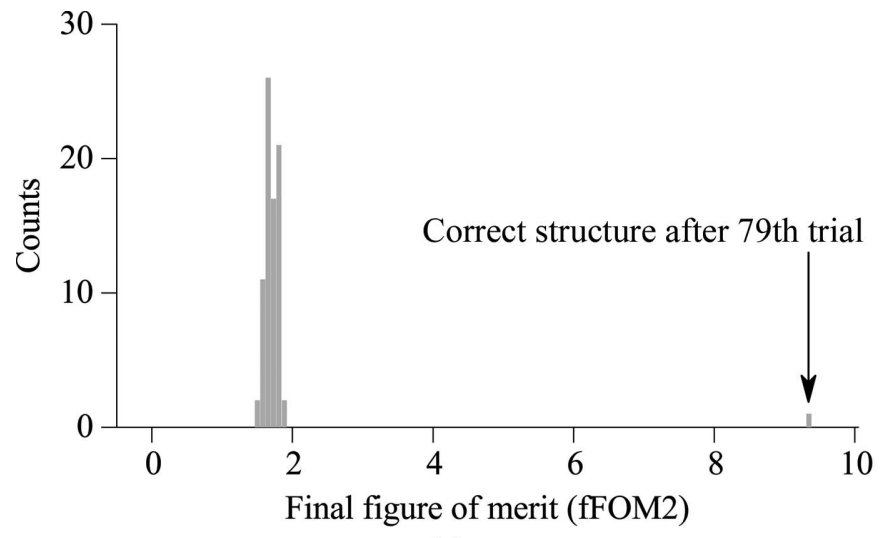

(a)

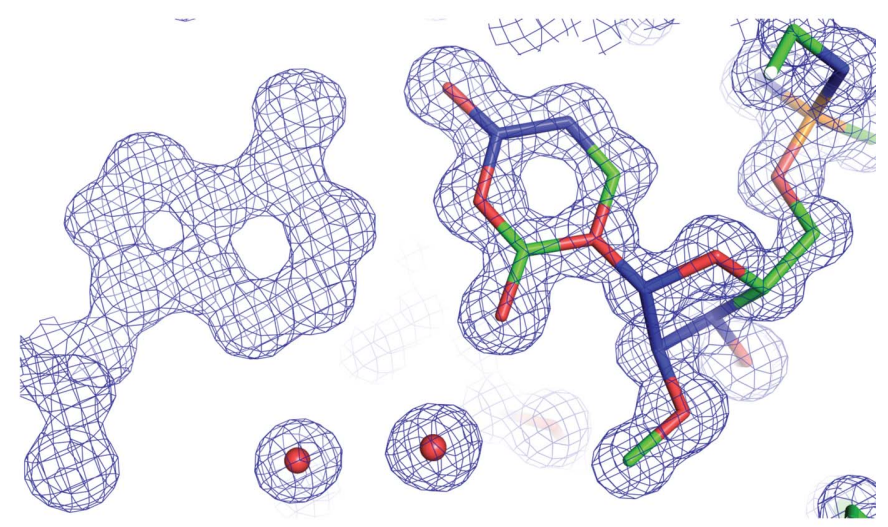

(b)

Figure 5

Initial structure determination of the known 27-nucleotide hairpin from random phases by direct methods using SIR2014. (a) Distribution of the fFOM2. (b) $F_{\mathrm{o}} \exp (\operatorname{SIR} 2014$ phases) map. 
model from Nautilus with the molecular-graphics program Coot and refined the rebuilt model with PHENIX using riding $\mathrm{H}$ atoms and anisotropic ADPs. The final structure had a MolProbity clash score of less than 1 (Chen et al., 2010). The final coordinates were deposited in the Protein Data Bank (Berman et al., 2000).

\subsection{Initial $a b$ initio structure determination of the hairpin RNA}

The above procedure was also used to determine the structure of the hairpin RNA. A correct structure was reached on the 79th trial in the initial phasing experiment (Fig. 5a). The fFOM2 for the correct structure was close to 10 and was almost twice the fFOM2 for the dsRNA (Fig. 1b). The mean of the fFOM2s for the failed trials was closer to 2.0 compared with the mean for the dsRNA (Fig. 1b). These differences in the distributions of the fFOM2s could have many causes including the absence of TPS, a slightly smaller asymmetric unit and somewhat higher resolution data for the hairpin. The $a b$ initio-phased electron-density map showed the methyl group on the $\mathrm{O} 2^{\prime}$-methyluridine at site 2650 of the hairpin
RNA (Fig. 5b). The light-atom assignment was also inaccurate in this structure.

\subsection{Distributions of the number of trials before success}

We repeated the phasing experiments 91 times with the dsRNA data (Fig. 6a) and 365 times with the hairpin data (Fig. 6c) to characterize the distribution of the number of failed trials before success. Each phasing experiment tried up to 600 sets of random phases. 600 trial phase sets could be tested within the $48 \mathrm{~h}$ time limit for the batch jobs running at OSCER. We tested 17070 different sets of random phases with the dsRNA data and 15384 different sets of random phases with the hairpin data. Of the 91 experiments initiated for the dsRNA, 68 phasing experiments led to correct structures (Fig. 6a). The arithmetic mean number of failed trials before the first successful trial was 239.4 (s.d. = 160.6). With the hairpin data, 364 of 365 phasing experiments led to correct structures (Fig. 6c). The mean number of failed trials before the successful trial with the hairpin data was 41.3 (s.d. $=41.5$ ). The geometric probability plots show that both sets of count data follow geometric distributions (Figs. $6 b$ and $6 d$ ). The

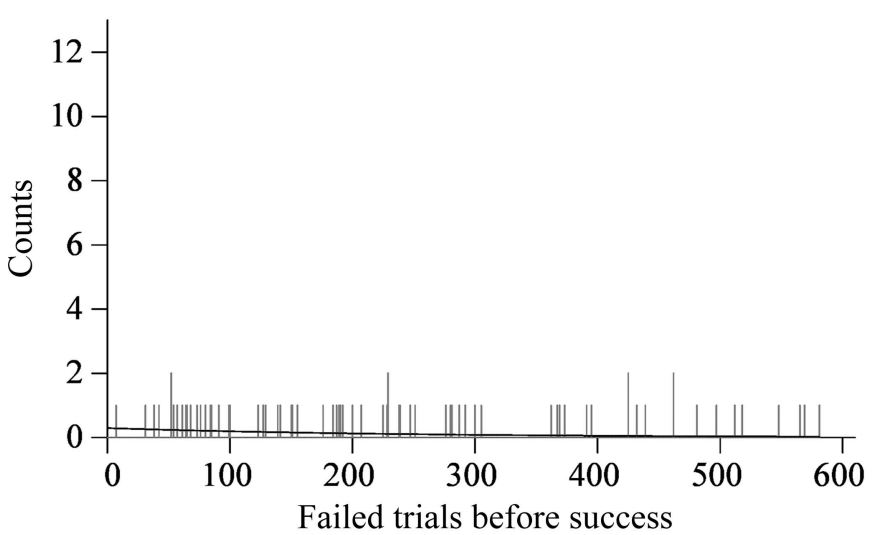

(a)

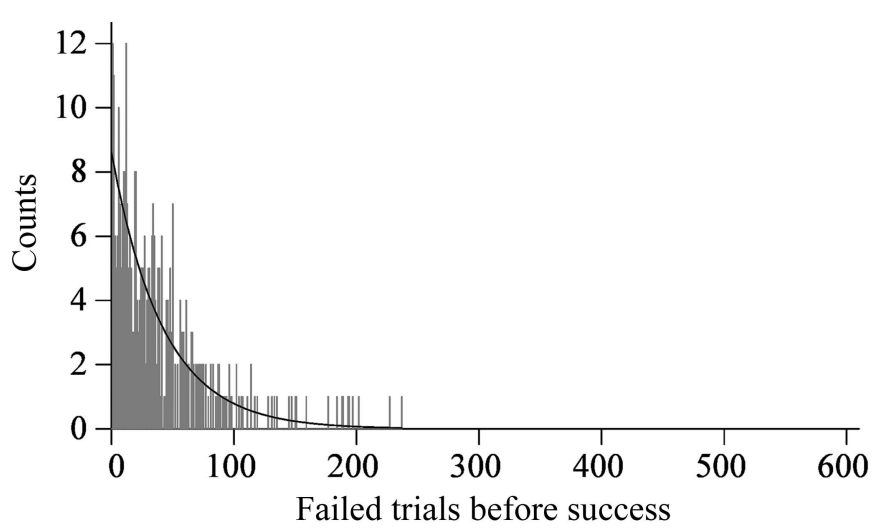

Figure 6

(c)

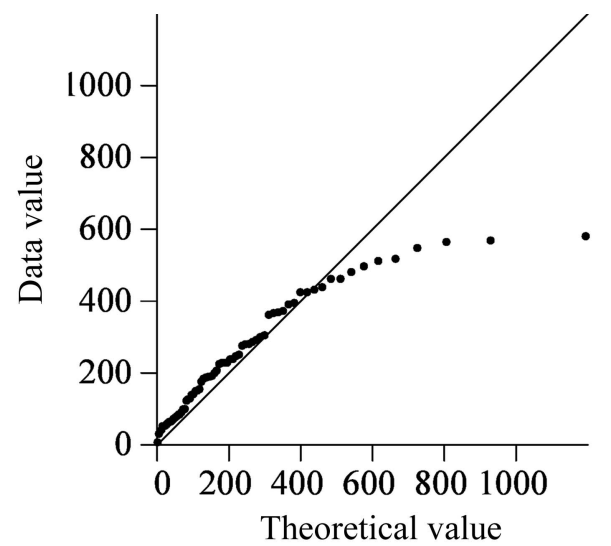

(b)

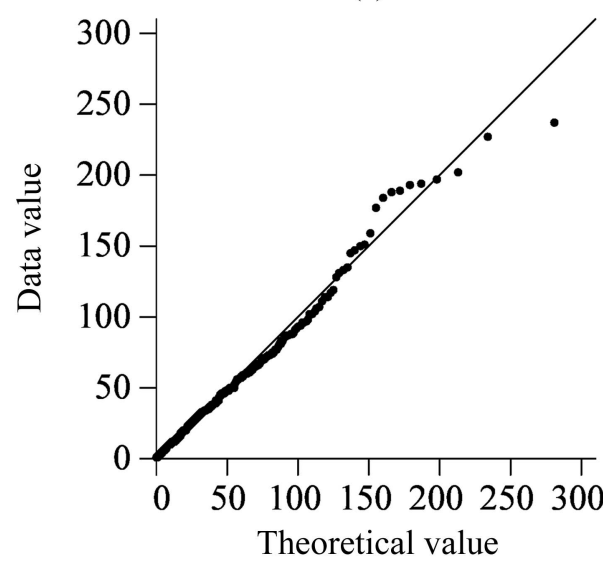

(d)

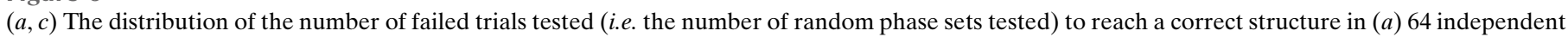

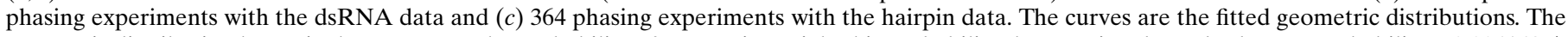

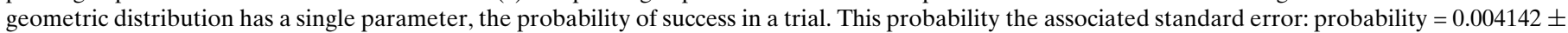

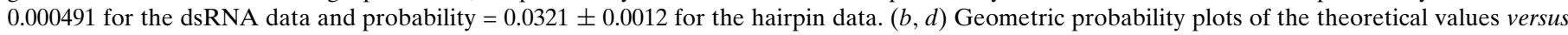

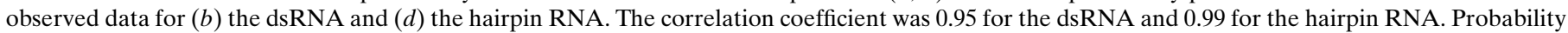
computations were performed with the MASS package in $R$ (Venables \& Ripley, 2002). 
outliers for the dsRNA in Fig. 6(b) are a result of limiting the number of trials to 600 . For clarity, the theoretical pmfs are shown as continuous curves instead of as impulse plots like the empirical pmfs (Figs. $6 a$ and $6 c$ ). The geometric distribution has a single parameter, the probability: $0.00414 \pm 0.000491$ for the dsRNA and $0.0231 \pm 0.0012$ for the hairpin. The two empirical pmfs were also compared with the nonparametric $K$-sample Anderson-Darling test (Scholz \& Stephens, 1987; Scholz \& Zhu, 2015), which makes no assumptions about the distribution for the random variable. The null hypothesis that all samples come from the same population was easily rejected $\left(p=2.3016 \times 10^{-43}\right)$.

\subsection{Comparison of intensity distributions}

The TPS of the dsRNA was expected to distort the distribution of the intensities. We compared the empirical cumulative distribution functions (cdfs) of the normalized structure factors squared (i.e. $\left|E^{2}\right|=Z$, acentric reflections only) from the observed data with the theoretical cdf for the Wilson distribution of acentric reflections. The acentric data from the hairpin followed the theoretical acentric distribution (Fig. 7b), but the cdf for the dsRNA data did not (Fig. 7a). The cdf for the dsRNA data also did not follow the theoretical distribution for perfect TPS with three repetitions (data not shown; Srinivasan \& Parathasarathy, 1976). This discrepancy may be

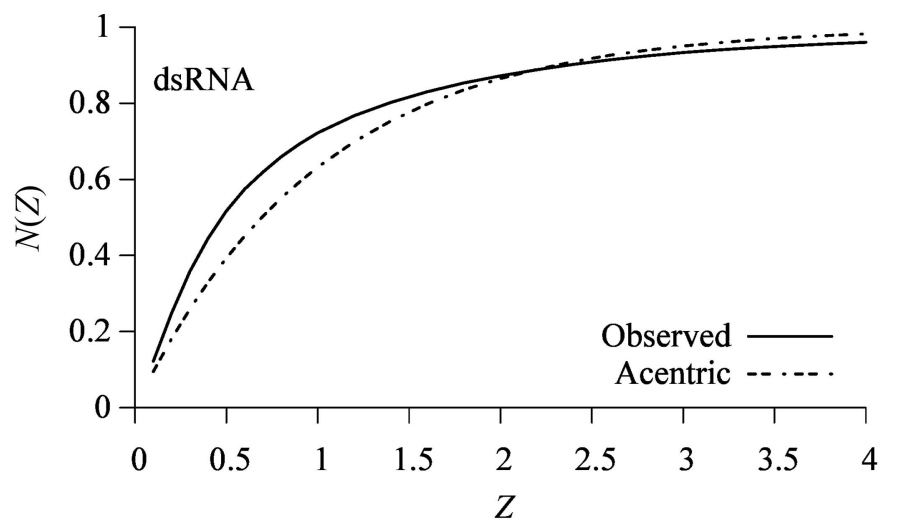

(a)

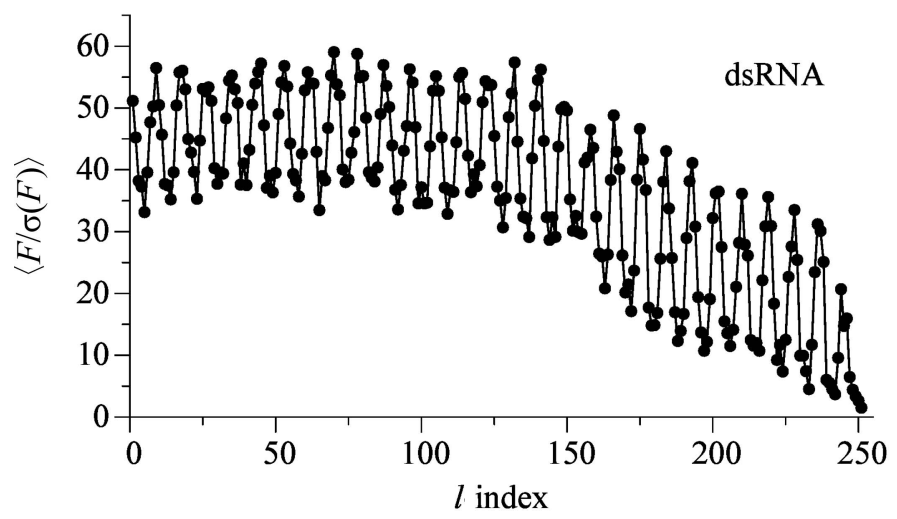

(c) caused by the imperfect TPS of the dsRNA. This imperfect TPS is reflected in the bimodal pattern of the mean structure factors when averaged by their $l$ index (Fig. 7c). The TPS enhanced the intensity of reflections with $l$ indices of $9 n$ or close to $9 n$ and depressed the intensity of reflections with values of $l=9 n \pm 4$ or 5 (Fig. 7c). The hairpin data lacked the alternating pattern in the amplitudes when averaged by their $l$ indices (Fig. 7d).

\subsection{Distribution of the RELAX vectors}

A correct structure often developed in the wrong position in the unit cell. The RELAX procedure in the SIR2014 procedure attempted to shift the structure to the proper position. The counts of the $x$ and $y$ components of the shift vectors were evenly distributed between the different origins in the $\mathbf{a} \times \mathbf{b}$ plane of both of the unit cells (data not shown). The $z$ coordinate was arbitrary for the hairpin in $P_{3}$. The $z$ components of the shift vectors for the dsRNA were zero (the correct value because the molecular dyad sits on a crystallographic twofold) for about one eighth of the trials but had different values for the remaining trials (Fig. 8). These nonzero values reflect the difficulty in placing the dsRNA along the $c$ axis in the presence of TPS.

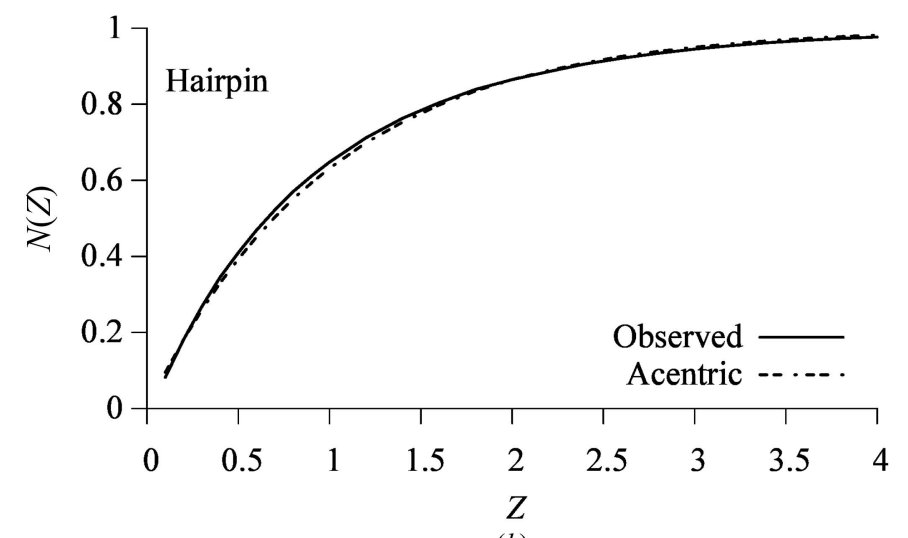

(b)

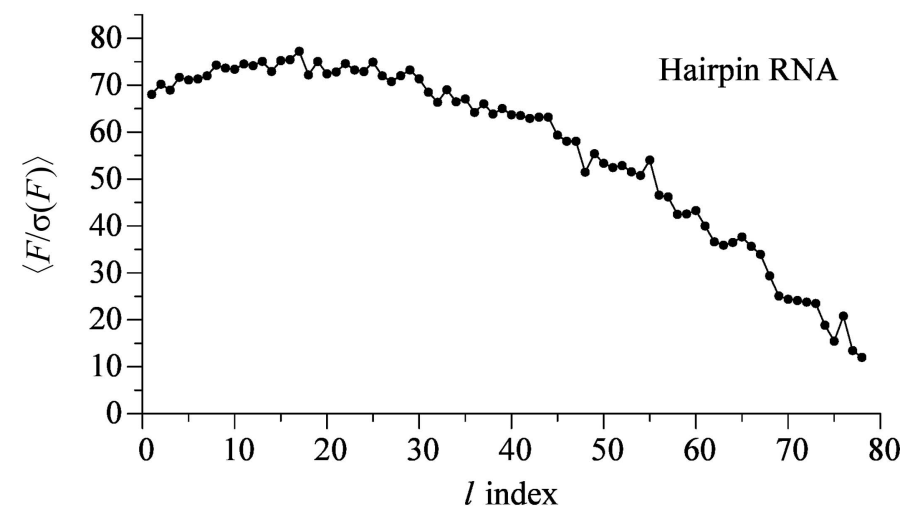

(d)

Figure 7

Intensity and structure-factor statistics. The cumulative distribution function (cdf) of the normalized intensities $\left(Z=E^{2}\right)$ for the observed data (solid lines) and the Wilson acentric distribution (dashed lines) for $(a)$ the dsRNA data and $(b)$ the hairpin data. $(c, d)$ Each structure factor was divided by its corrected sigma and then averaged by its $l$ Miller index. $(c)$ The diffraction data for the dsRNA; $(d)$ the diffraction data for the hairpin RNA. 


\subsection{Removal of the strongest reflections}

The TPS in the dsRNA data caused the strongest reflections to contribute more to the total scattering power than in the hairpin RNA data (Fig. 9). Structure determinations with the dsRNA data were expected to be more sensitive to the loss of the strongest reflections. Removal of the top 81 reflections from the dsRNA had the same effect as removing the top 232 reflections from the hairpin data (Fig. 10). The strongest reflections were more important in the dsRNA data.

\section{Discussion}

4.1. Structure determination from random phases in the presence of imperfect TPS

We report a case of $a b$ initio structure determination of a dsRNA with TPS caused by the helical repeats. This intramolecular TPS was strong enough to give a bimodal structurefactor distribution but was too weak to be automatically detected by SIR2014. We compare this case with that of a

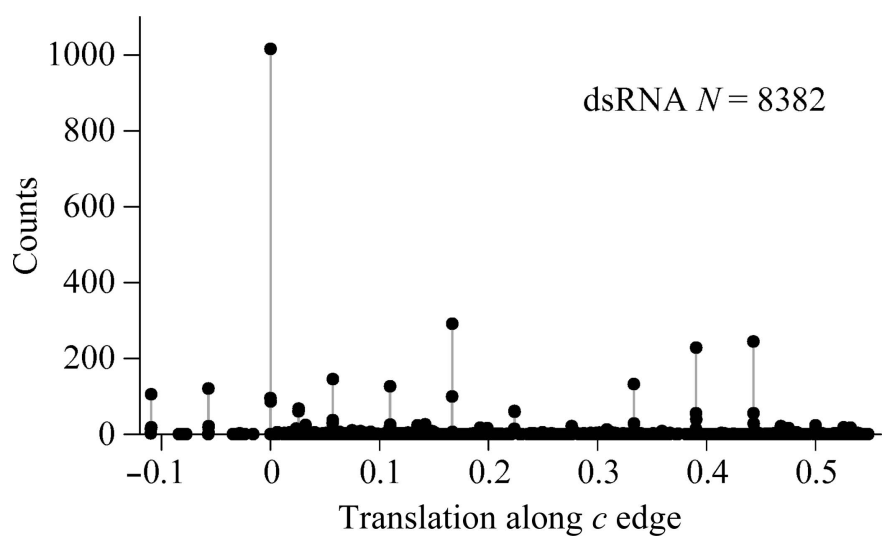

Figure 8

Distribution of the $z$ components of the SIR2014 RELAX shift vectors for moving misplaced trial structures to the correct origin in phasing experiments with the dsRNA data in the presence of TPS.

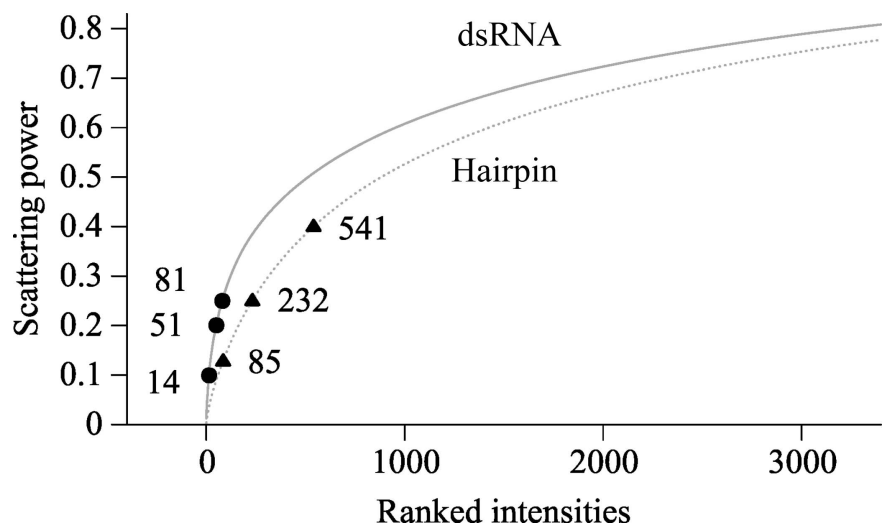

Figure 9

Cumulative scattering power of the diffraction data from the dsRNA (solid line) and hairpin RNA (dashed line). The quotient of the structure factor squared and the sum of the squared structure factors gave the relative contribution of a particular reflection to the total scattering power. The contribution of $F_{000}$ was ignored. The points indicate the numbers of strong reflections removed in deletion data sets that tested the importance of the strongest reflections in phasing experiments.
RNA hairpin of similar size and with data of similar resolution but without TPS. Success with the dsRNA (675 non-H atoms) was achieved in $10 \mathrm{~h}$ using one CPU. This result was obtained with $1.05 \AA$ resolution diffraction data from a crystal with no atoms heavier than phosphorus. We found no published evidence of a $a b$ initio direct-methods structure determination of a larger nucleic acid in the absence of calcium or heavier atoms. The dsRNA is $41 \%$ larger than the previous record (Table 3).

\subsection{Intramolecular TPS in RNA with three helical turns}

TPS usually relates copies of biopolymers within the asymmetric unit (Zwart et al., 2008), but here the TPS relates helical repeats in one strand of the dsRNA. This intramolecular TPS was imperfect owing to 'displacive' deviations in atom positions from ideal pseudosymmetry and 'replacive' deviations in atomic composition owing to differences in sequence and termini structure (MacKay, 1953; Cascarano et al., 1988a,b). The partial TPS caused high valleys in the

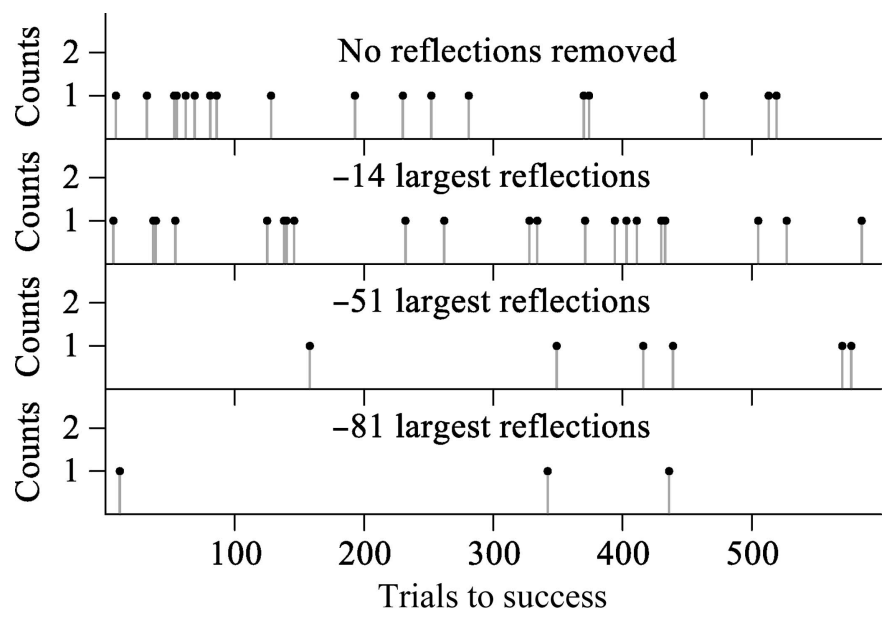

(a)

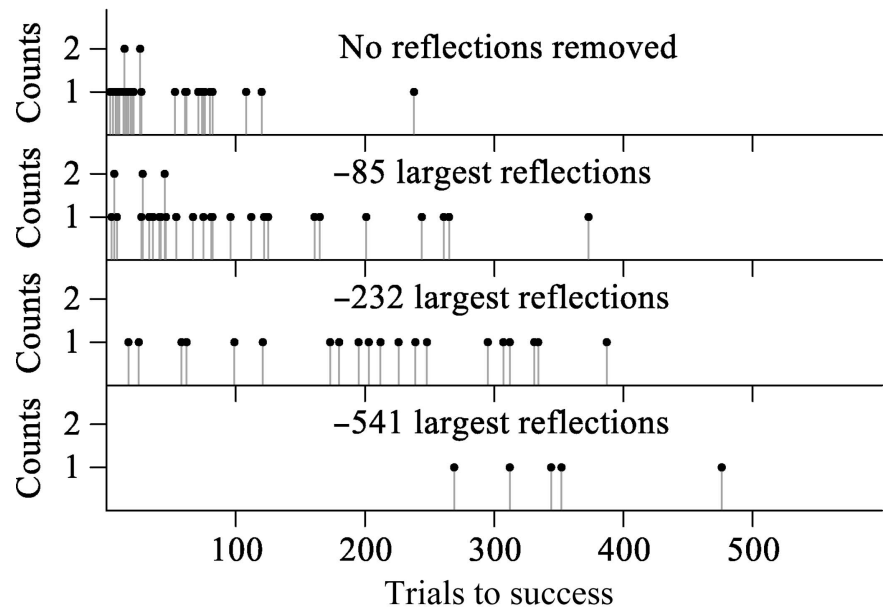

(b)

Figure 10

Comparison of the phasing experiments with all of the data and three deletion data sets. Each data set was used in 30 phasing experiments. (a) With the dsRNA (PDB entry 5da6), there were 30, 20, seven and four successes from top to bottom. (b) With the hairpin (PDB entry 5d99), there were $30,30,20$ and five successes from top to bottom. 
Table 3

Previously unknown nucleic acid crystal structures determined by direct methods starting from random phases.

\begin{tabular}{lllllll}
\hline Molecule & Non-H $\dagger$ & $d_{\min }(\AA)$ & $Z>19$ & Program & PDB code & Reference \\
\hline 10 nt DNA & 408 & 0.83 & 0 & SnB & 1dpl & Egli et al. $($ (1998) \\
11 nt DNA/RNA & 414 & 1.15 & $3 \mathrm{Ca}^{2+}$ & SnB & Han $(2001)$ & Safaee et al. $(2013)$ \\
11 nt RNA & 478 & 1.0 & 0 & SHELXD & 4jrd & Luo et al. $(2014)$ \\
12 nt DNA & 243 & 0.75 & 0 & SHELXD & 4ocb & This work \\
32 nt RNA & 675 & 1.05 & 0 & SIR2014 & 5da6 \\
\hline
\end{tabular}

$\uparrow$ The number of non-H nucleic acid atoms in the asymmetric unit.

bimodal structure factors averaged by their $l$ Miller indices. More sensitive methods are needed for the automated detection of partial TPS.

\subsection{TPS enhanced the role of the strongest reflections in phasing}

The largest $E$ values give the most reliable phase relationships. The loss of only the top $1 \%$ reduced the number of successful phasing experiments with both data sets, and the dsRNA data were more sensitive to the loss of the strongest reflections than the hairpin data. The dynamic range of the diffraction intensities can be $10^{7}$ from crystals of dsRNA, so detector saturation is a serious issue. Guidelines for collecting complete diffraction data at atomic resolution can be found in Dauter (1999).

\subsection{The number of trials to a correct structure}

Structure determination by $a b$ initio direct methods is a stochastic process, so we repeated the phasing experiments large numbers of times $(n>90)$ for both data sets to obtain the empirical probability mass functions (pmfs) of the number of failed trials before success. The mean number of trials for the dsRNA was nearly six times larger than that for the hairpin. Both pmfs have geometric distributions, in agreement with the probability theory for Bernoulli trials, but the pmfs were statistically different in spite of similar completeness, resolution limit and size. Phasing experiments with other 32-basepair RNAs are needed to to determine whether the pmf for the diffraction data from the dsRNA is representative of the pmfs for other 32-base-pair RNAs in the same space group. This requirement also applies to hairpin RNAs. Nonetheless, our pmfs provide benchmarks for hard and easy structure determinations of nucleic acids by $a b$ initio direct methods.

\subsection{Other $a b$ initio structure-determination protocols}

Other direct-methods programs [e.g. SHELXD (Sheldrick, 2008) and SnB (Miller et al., 2007)] use different phasing protocols. One or more of these programs may also succeed with the dsRNA data. The charge-flipping program SUPERFLIP succeeded with a case of a planar molecule of 45 atoms with intermolecular TPS (Oszlányi et al., 2006), but we had no success with the dsRNA data. The programs $A R P / w A R P$ and $A C O R N$ can start phasing in real space with randomly placed atoms (Tame, 2000; Dodson \& Woolfson, 2009), but ACORN failed with the dsRNA data when starting from a random atom. Success where failure occurred may still be possible by optimization of the parameters of a protocol.

\section{Acknowledgements}

We thank Dr Tzanko Doukov for help with data collection at Stanford Synchrotron Radiation Lightsource (SSRL) beamline 7-1. The Structural Molecular Biology Program at SSRL is supported by DOE-OBER, NIH-NCRR (P41RR001209) and NIH-NIGMS. We thank Drs Gianluca Cascarano and Sabino Maggi for help with installing beta versions of SIR2011 and SIR2014, Dr Henry Neeman and Josh Alexander for installing SIR2011 and SIR2014 on the OSCER supercomputer, Dr George Sheldrick for help with calculation of the mean phase error and Chiedza Kanyumbu and Kathy Kyler for editing the manuscript. This work was supported in part by grants from the Oklahoma Center for the Advancement of Science and Technology (OCAST HR08-138) and by a grant from the NIH/National Institute of Allergies and Infectious Diseases (R01 AI088011; PI: Blaine Mooers). The research reported in this publication was also supported in part by an Institutional Development Award (IDeA) from the National Institute of General Medical Sciences of the National Institutes of Health under grant No. P20 GM103640.

\section{References}

Adams, P. D. et al. (2010). Acta Cryst. D66, 213-221.

Battye, T. G. G., Kontogiannis, L., Johnson, O., Powell, H. R. \& Leslie, A. G. W. (2011). Acta Cryst. D67, 271-281.

Berman, H. M., Westbrook, J., Feng, Z., Gilliland, G., Bhat, T. N., Weissig, H., Shindyalov, I. N. \& Bourne, P. E. (2000). Nucleic Acids Res. 28, 235-242.

Böhme, R. (1982). Acta Cryst. A38, 318-326.

Burla, M. C., Caliandro, R., Carrozzini, B., Cascarano, G. L., Cuocci, C., Giacovazzo, C., Mallamo, M., Mazzone, A. \& Polidori, G. (2015). J. Appl. Cryst. 48, 306-309.

Burla, M. C., Caliandro, R., Carrozzini, B., Cascarano, G. L., De Caro, L., Giacovazzo, C., Polidori, G. \& Siliqi, D. (2006). J. Appl. Cryst. 39, 527-535.

Burla, M. C., Carrozzini, B., Cascarano, G. L., Giacovazzo, C. \& Polidori, G. (2000). J. Appl. Cryst. 33, 307-311.

Burla, M. C., Carrozzini, B., Cascarano, G. L., Giacovazzo, C. \& Polidori, G. (2002). Z. Kristallogr. 217, 629-635.

Burla, M. C., Giacovazzo, C. \& Polidori, G. (2013). J. Appl. Cryst. 46, 1592-1602.

Caliandro, R., Carrozzini, B., Cascarano, G. L., De Caro, L., Giacovazzo, C., Mazzone, A. \& Siliqi, D. (2008). J. Appl. Cryst. 41, 548-553.

Caliandro, R., Carrozzini, B., Cascarano, G. L., De Caro, L., Giacovazzo, C. \& Siliqi, D. (2007). J. Appl. Cryst. 40, 931-937. 
Cascarano, G., Giacovazzo, C. \& Luić, M. (1985). Acta Cryst. A41, 544-551.

Cascarano, G., Giacovazzo, C. \& Luić, M. (1987). Acta Cryst. A43, $14-22$.

Cascarano, G., Giacovazzo, C. \& Luić, M. (1988a). Acta Cryst. A44, 176-183.

Cascarano, G., Giacovazzo, C. \& Luić, M. (1988b). Acta Cryst. A44, 183-188.

Chen, V. B., Arendall, W. B., Headd, J. J., Keedy, D. A., Immormino, R. M., Kapral, G. J., Murray, L. W., Richardson, J. S. \& Richardson, D. C. (2010). Acta Cryst. D66, 12-21.

Cowtan, K. (2014). IUCrJ, 1, 387-392.

Dauter, Z. (1999). Acta Cryst. D55, 1703-1717.

Dauter, Z., Botos, I., LaRonde-LeBlanc, N. \& Wlodawer, A. (2005). Acta Cryst. D61, 967-975.

Dodson, E. J. \& Woolfson, M. M. (2009). Acta Cryst. D65, 881-891.

Egli, M., Tereshko, V., Teplova, M., Minasov, G., Joachimiak, A., Sanishvili, R., Weeks, C. M., Miller, R., Maier, M. A., An, H., Cook, P. D. \& Manoharan, M. (1998). Biopolymers, 48, 234-252.

Emsley, P., Lohkamp, B., Scott, W. G. \& Cowtan, K. (2010). Acta Cryst. D66, 486-501.

Evans, P. (2006). Acta Cryst. D62, 72-82.

Fan, H.-F., Qian, J.-Z., Yao, J.-X., Zheng, C.-D. \& Hao, Q. (1988). Acta Cryst. A44, 691-692.

Fan, H.-F., Yao, J.-X. \& Qian, J.-Z. (1988). Acta Cryst. A44, 688-691.

Giacovazzo, C. (1998). Direct Phasing in Crystallography. Oxford University Press.

Giacovazzo, C. (2014). Phasing in Crystallography: A Modern Perspective. Oxford University Press.

Gramlich, V. (1984). Acta Cryst. A40, 610-616.

Han, G. W. (2001). Acta Cryst. D57, 213-218.

Hauptman, H. \& Karle, J. (1959). Acta Cryst. 12, 846-850.

Hubbard, S. R., Greenall, R. J. \& Woolfson, M. M. (1994). Acta Cryst. D50, 833-841.

Keating, K. S. \& Pyle, A. M. (2012). Acta Cryst. D68, 985-995.

Kondo, J., Urzhumtseva, L. \& Urzhumtsev, A. (2008). Acta Cryst. D64, 1078-1091.

Kumar, K. S. D., Gurusaran, M., Satheesh, S. N., Radha, P., Pavithra, S., Thulaa Tharshan, K. P. S., Helliwell, J. R. \& Sekar, K. (2015). J. Appl. Cryst. 48, 939-942.

Langs, D. A. \& Hauptman, H. A. (2011). Acta Cryst. A67, 396-401. Lunin, V. Y. \& Woolfson, M. M. (1993). Acta Cryst. D49, 530-533.
Luo, Z., Dauter, M. \& Dauter, Z. (2014). Acta Cryst. D70, 1790-1800. Mackay, A. L. (1953). Acta Cryst. 6, 214-215.

Miller, R., Shah, N., Green, M. L., Furey, W. \& Weeks, C. M. (2007). J. Appl. Cryst. 40, 938-944.

Mooers, B. H. M. \& Matthews, B. W. (2004). Acta Cryst. D60, 17261737.

Mooers, B. H. M. \& Matthews, B. W. (2006). Acta Cryst. D62, 165176.

Mooers, B. H. M. \& Singh, A. (2011). RNA, 17, 1870-1883.

Moriarty, N. W., Grosse-Kunstleve, R. W. \& Adams, P. D. (2009). Acta Cryst. D65, 1074-1080.

Morris, R. J. \& Bricogne, G. (2003). Acta Cryst. D59, 615-617.

Myers-Turnbull, D., Bliven, S. E., Rose, P. W., Aziz, Z. K., Youkharibache, P., Bourne, P. E. \& Prlić, A. (2014). J. Mol. Biol. 426, 2255-2268.

Olieric, V., Rieder, U., Lang, K., Serganov, A., Schulze-Briese, C., Micura, R., Dumas, P. \& Ennifar, E. (2009). RNA, 15, 707-715.

Oszlányi, G., Sütő, A., Czugler, M. \& Párkányi, L. (2006). J. Am. Chem. Soc. 128, 8392-8393.

Parkinson, G., Vojtechovsky, J., Clowney, L., Brünger, A. T. \& Berman, H. M. (1996). Acta Cryst. D52, 57-64.

Ramagopal, U. A., Dauter, M. \& Dauter, Z. (2003). Acta Cryst. D59, 1020-1027.

Read, R. J., Adams, P. D. \& McCoy, A. J. (2013). Acta Cryst. D69, $176-183$.

Safaee, N., Noronha, A. M., Rodionov, D., Kozlov, G., Wilds, C. J., Sheldrick, G. M. \& Gehring, K. (2013). Angew. Chem. Int. Ed. 52, 10370-10373.

Scholz, F. W. \& Stephens, M. A. (1987). J. Am. Stat. Assoc. 82, 918-924.

Scholz, F. \& Zhu, A. (2015). kSamples: K-sample Rank Tests and their Combinations. https://cran.r-project.org/web/packages/kSamples/ index.html.

Sheldrick, G. M. (1990). Acta Cryst. A46, 467-473.

Sheldrick, G. M. (2008). Acta Cryst. A64, 112-122.

Srinivasan, R. \& Parathasarathy, S. (1976). Some Statistical Applications in $X$-ray Crystallography. Oxford: Permagon Press.

Tame, J. R. H. (2000). Acta Cryst. D56, 1554-1559.

Venables, W. N. \& Ripley, B. D. (2002). Modern Applied Statistics with $S$, 4th ed. New York: Springer.

Zwart, P. H., Grosse-Kunstleve, R. W., Lebedev, A. A., Murshudov, G. N. \& Adams, P. D. (2008). Acta Cryst. D64, 99-107. 